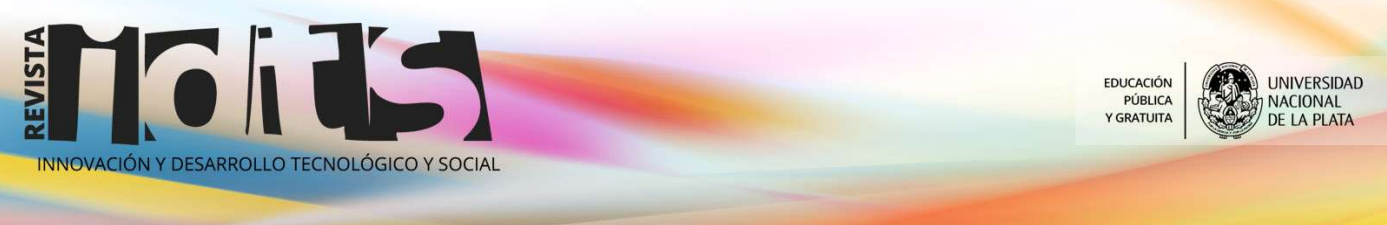

Innovación y Desarrollo Tecnológico y Social (2019) 1 (1): 41-51

\title{
Herramienta informática para la identificación de especies de serpientes bonaerenses
}

\author{
La Frazia Luciano1, Laura Lanzarini, César Estrebou², Luis Giambelluca³ \\ ${ }^{1}$ Facultad de Informática. Universidad Nacional de La Plata \\ ${ }^{2}$ Instituto de Investigación en Informática LIDI. Facultad de Informática. UNLP \\ ${ }^{3}$ Centro de Estudios Parasitológicos y de Vectores (CEPAVE). CONICET La Plata. UNLP \\ ${ }^{4}$ laural@lidi.info.unlp.edu.ar
}

\begin{abstract}
Resumen. Sin bien las serpientes cumplen una actividad de relevancia en el control de ciertas plagas, se considera que deben ser eliminadas con celeridad, ya que sólo pueden causar problemas a la población. Afortunadamente, en la actualidad, se comenzó a rever esa postura, dándole a las serpientes un rol mucho más racional, considerándolas como otro grupo que conforma un ecosistema. El desarrollo de este proyecto busca resguardar las distintas especies de serpientes de la Provincia de Buenos Aires y en particular las del partido de La Plata, así como concientizar y educar a los usuarios sobre los distintos ofidios que habitan la zona. Resultará de suma utilidad para aquellas personas que desempeñen un rol tanto en la salud (salas de primeros auxilios, hospitales) o de seguridad (bomberos, policías); grupos que muchas veces deben decidir qué hacer con un ejemplar hallado en un ámbito no esperado o qué medidas tomar respecto a una persona posiblemente intoxicada por una mordedura.

El desarrollo realizado corresponde a una aplicación informática que, a partir de la foto de un ejemplar, posee la capacidad de informar a qué especie corresponde junto con su grado de peligrosidad. El proceso inicia con la captura de la imagen de un ejemplar de serpiente. Una vez cargada la foto, el sistema identifica las zonas de la imagen con mayor información, las cuales, generalmente, se relacionan con el color, la textura y el diseño de las manchas del animal. Esta información del ejemplar se la compara con una base de datos formada por las distintas especies presentes en la zona, para determinar de qué tipo de especie de serpiente se trata. Como devolución, el usuario recibe un resumen detallado sobre las particularidades de ese animal y cuál es su riesgo sanitario, junto con el grado de acierto (credibilidad de la respuesta). Si bien existen soluciones equivalentes para otros tipos de organismos, como por ejemplo plantas, y otras especies animales, esta es la primera aplicación dedicada a esta temática acerca de ejemplares de la zona.
\end{abstract}

Ofidios; Herpetología; Protección de animales, Procesamiento de imágenes; Visión artificial; Reconocimiento de objetos.

Recibido: 10/04/2019 Aceptado: 17/07/2019

DOI: https://doi.org/10.24215/26838559e003 


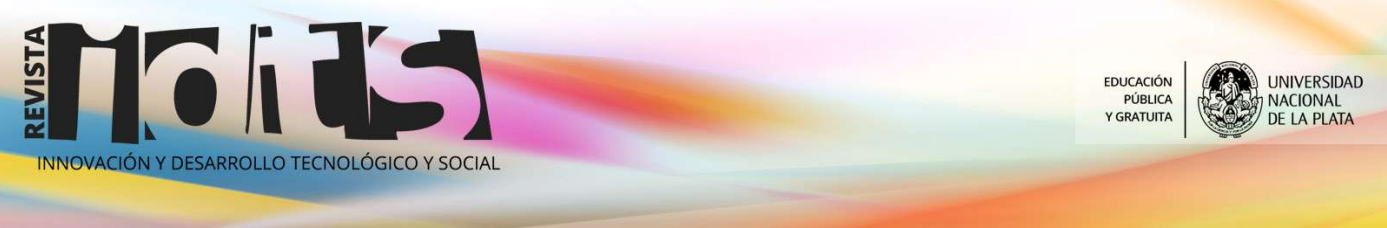

Innovación y Desarrollo Tecnológico y Social (2019) 1 (1): 41-51

\title{
Software for the identification of species of snakes from Buenos Aires Province
}

\begin{abstract}
Although snakes have an important role in the control of certain pests it is considered that they should be eliminated quickly because they can only cause problems to people. Fortunately, at present, this position has begun to be reviewed, giving snakes a much more rational role, considering them as another group among other species that conform an ecosystem. The development of this project aims to protect the various species of snakes in Province of Buenos Aires and in particular those of $L a$ Plata district, as well as to raise awareness and educate users about the different ophidians that inhabit the area. It will be extremely useful for those who play a role in health (first aid rooms, hospitals) or safety (firefighters, police); groups that often have to decide what to do with a specimen found in an unexpected environment or how to proceed when a person is poisoned by a bite.

The development carried out corresponds to a computer application that starting with a photograph of a specimen can inform the species to which they belong and its degree of danger. The process begins with the capture of the image of a snake specimen. Once the photograph is uploaded, the system identifies areas of the image with relevant information, which are generally related to the color, texture and design of the animal's spots. Using this information, the specimen is compared with a database formed by different species present in the area, to determine its type. In response, the user receives a detailed summary of the particularities of that animal, its health risk and the degree of success (credibility of the response).

Although there are equivalent solutions for other types of organisms, such as plants and other animal species, this is the first application dedicated to this subject to specimens from the area.

Ophidia; Herpetology; Animal protection; Image processing; Artificial vision; Object recognition.
\end{abstract}




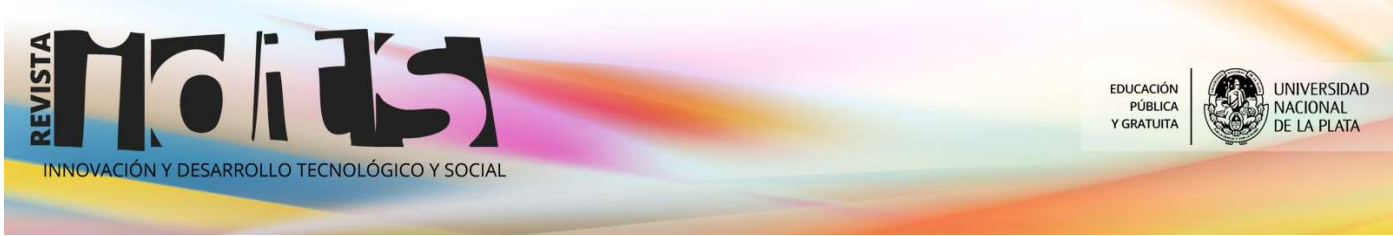

Innovación y Desarrollo Tecnológico y Social (2019) 1 (1): 41-51

\section{Novedad u originalidad local en el conocimiento}

El reconocimiento de objetos en imágenes por medio de aplicaciones informáticas constituye una parte fundamental del área conocida como Visión por Computadora y es una herramienta sumamente útil en distintos contextos tales como reconocimiento de rostros, detección vehículos, conteo de peatones e incluso, como en este caso, para identificación de distintos tipos de serpientes.

Si bien existen artículos donde se propone reconocer especies de serpientes utilizando técnicas de aprendizaje automático (Amiza et al., 2017), el principal aporte radica en el procesamiento de imágenes de especies de serpientes bonaerenses. A la fecha, los autores de este trabajo no tienen conocimiento de la existencia de aplicaciones de software similares.

\section{Grado de relevancia}

La aplicación informática desarrollada constituye una herramienta de prevención sumamente útil para humanos y ofidios. Se espera que las personas, manteniendo una distancia prudencial del ejemplar, logren tener conocimiento del grado de peligrosidad de la serpiente en cuestión y sólo decidan eliminarla en caso de que sea estrictamente necesario. Por otro lado, en caso de tener conocimiento de la existencia de ejemplares de serpientes peligrosos en la zona, será posible alertar a los centros de salud para que arbitren las medidas sanitarias del caso.

Actualmente, la aplicación desarrollada se ejecuta en una computadora de escritorio dado que requiere contar con información almacenada de las especies a reconocer. Su ejecución en dispositivos móviles (teléfonos celulares o tablets) requiere de un servicio de almacenamiento remoto ya sea basado en un servidor específico o a través de un servicio en la nube (cloud). A futuro, se espera que el usuario capture la imagen del ejemplar con su dispositivo y obtenga la información correspondiente de manera inmediata.

\section{Grado de pertinencia}

La aplicación desarrollada a partir de las investigaciones realizadas tiene la capacidad de efectuar el reconocimiento esperado. Es decir que resuelve el problema identificando algunas de las especies más frecuentes de la zona del partido de La Plata.

Desde el punto de vista informático, su implementación involucró el diseño y desarrollo de una base de datos de serpientes bonaerenses inexistente hasta el momento. Esto es un aporte sumamente útil ya que es un insumo fundamental para desarrollar otros clasificadores equivalentes y comparar su desempeño con el aquí propuesto.

Como herramienta educativa, la aplicación desarrollada puede utilizarse para familiarizar a la población con las especies de serpientes que se encuentran en la zona. En caso de no contar con un ejemplar para fotografiar, podría utilizarse un muestrario de ejemplares previamente almacenados del cual sea posible seleccionar una imagen al azar y obtener la información correspondiente. 


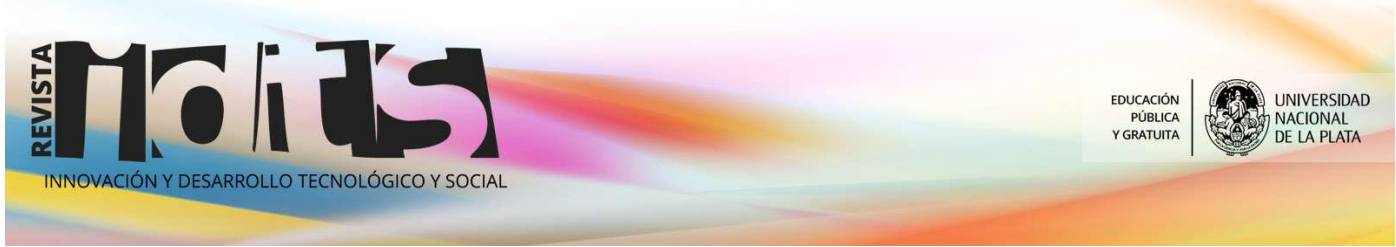

Innovación y Desarrollo Tecnológico y Social (2019) 1 (1): 41-51

\section{Grado de demanda}

Esta aplicación fue desarrollada como respuesta a la propuesta planteada por el centro de investigaciones científicas CEPAVE (Centro de Estudios Parasitológicos y de Vectores), dependiente del CONICET. El centro tiene como objetivo realizar investigación científica sobre la biología y ecología de parásitos, parasitoides, depredadores y patógenos de invertebrados y vertebrados de importancia sanitaria y económica. Según sus experiencias, en la actualidad, los bomberos voluntarios utilizan el libro Serpiente Bonaerenses (Giambelluca, 2015) para identificar posibles ofidios con los que puedan encontrarse en casos de incendios donde el animal tiende a aparecer muy frecuentemente.

\section{Desarrollo del producto}

El desarrollo inició con un estudio de los distintos tipos de serpientes bonaerenses y en particular con la identificación de las que pueden hallarse habitualmente en el partido de La Plata. Se hizo una descripción de la distribución de las distintas especies llegando a la conclusión de que la diversidad de serpientes en el partido de la Plata es más reducida que la del norte de la provincia de Buenos Aires, lo que facilitó la tarea de reconocimiento de las diferentes especies. Esto simplificó el desarrollo de la aplicación a través de una drástica reducción la cantidad de especies a analizar.

Una vez identificadas las especies a reconocer fue preciso construir una base de datos contra la cual comparar los distintos ejemplares a reconocer. Un aspecto importante a considerar fue la manera de representación a utilizar ya que se esperaba que la respuesta de la aplicación no dependiera de la manera en que la imagen hubiera sido captada. El método de representación elegido fue SIFT, definido por Lowe, capaz de extraer características de una imagen, las cuales pueden utilizarse para hallar correspondencia entre dos vistas distintas de un mismo objeto (Lowe, 2004). Dichas características, denominadas características SIFT (Scale Invariant Feature Transform), son invariantes a la escala y a la rotación de la imagen y bastante invariantes a distorsiones afines (affine distortion) así como a cambios del punto de vista y de iluminación. Además, poseen la capacidad de ser muy distintivas.

El proceso para determinar las características SIFT de una imagen consiste en cuatro pasos:

- $\quad$ En primer lugar, se calcula la ubicación de potenciales puntos de interés dentro de la imagen, los que corresponden a los puntos extremos, calculados a partir de subconjuntos de planos de diferencias de filtros gaussianos (DoG) aplicados sobre la imagen a distintas escalas.

- Luego, se descartan los puntos de interés que poseen bajo contraste. Esto es una mejora con respecto a lo definido en (Lowe, 1999).

- En tercer lugar, se calculan las orientaciones de los puntos de interés relevantes.

- Utilizando las orientaciones anteriores, se analiza el entorno de cada punto y se determina el vector de características correspondientes. 
Innovación y Desarrollo Tecnológico y Social (2019) 1 (1): 41-51

Como resultado de este proceso se obtiene un conjunto de descriptores, cada uno formado por 128 características, que pueden ser comparados con los correspondientes a otra imagen del mismo objeto con diferente escala, orientación y/o punto de vista.Dicha comparación puede realizarse directamente a través de una medida de distancia estableciendo un umbral de similitud.Para más detalles con respecto a este método se recomienda consultar (Lowe, 2004).

\section{Modelo desarrollado}

El desempeño de una aplicación informática capaz de reconocer un objeto está fuertemente relacionado con las características de dicho objeto que utilice para llevar a cabo esta tarea. En el caso de las especies de serpientes, una primera posibilidad podría ser utilizar las características más distintivas, tales como el color y la forma de su pupila o las escamas de su cabeza. La Figura 1 resume las características más relevantes de las especies de interés en este trabajo.

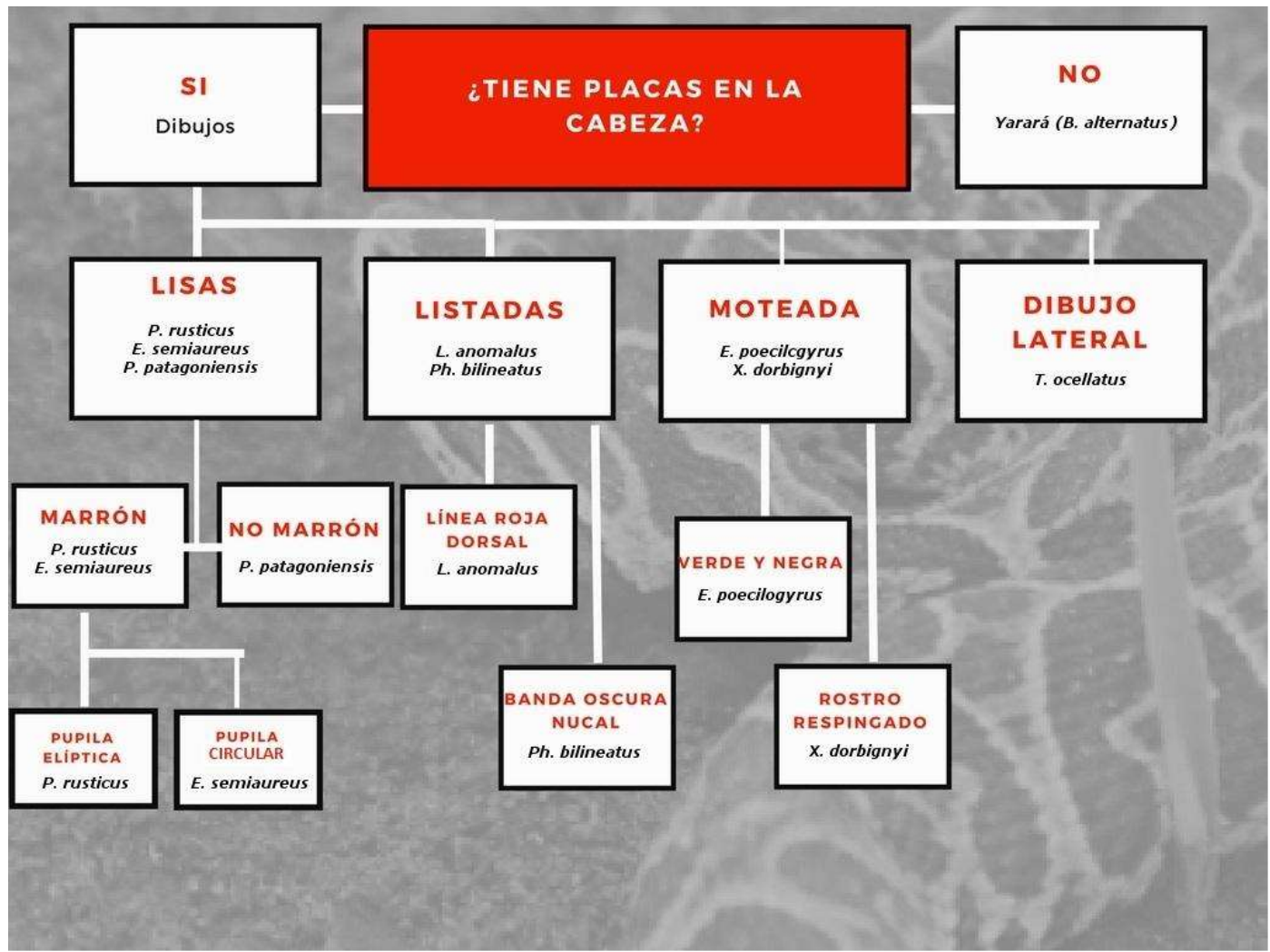

Figura 1. Árbol de decisión donde se detallan las características de cada especie/ Figure 1. Decision tree detailing the characteristics of each species

Si bien parece sencillo ejecutar un árbol como el de la figura 1, el usuario no siempre está en condiciones de responder muchas de las opciones que determinan la respuesta final. Por ejemplo, una persona sin conocimientos sobre serpientes no será capaz de diferenciar entre lisas y listadas. Por otro lado, suponiendo un encuentro casual con un ejemplar, las probabilidades de obtener una foto de su pupila o de las escamas de su 


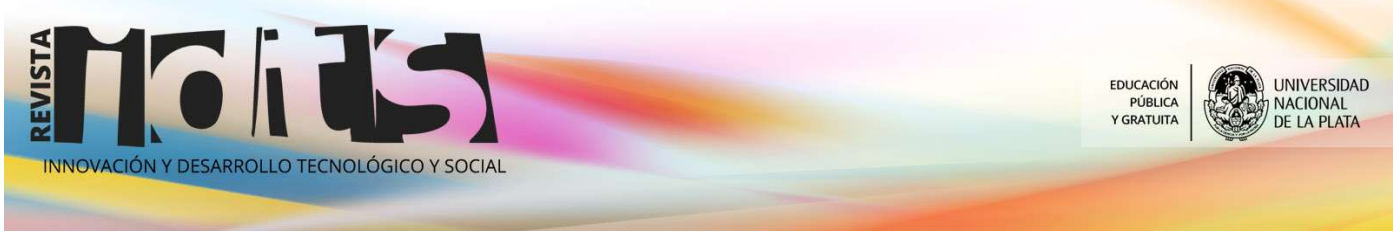

Innovación y Desarrollo Tecnológico y Social (2019) 1 (1): 41-51

cabeza son casi nulas, teniendo en cuenta que una persona sin conocimientos del tema, consideraría un peligro acercarse al animal. Además, resulta complejo obtener una foto de cuerpo entero de un ejemplar debido a que los lugares usuales de encuentro no son óptimos para conseguir una buena captura. Además, en ocasiones, las serpientes se encuentran enrolladas, ocultando partes de su cuerpo.

Por lo tanto, el modelo propuesto en este artículo no utiliza el árbol de la Figura 1 sino que se basa en el reconocimiento automático de las texturas de la región dorsal de los ofidios. El reconocimiento de un ejemplar consta de los siguientes pasos:

- Se captura la imagen del ejemplar a reconocer.

- $\quad$ Se calculan los descriptores SIFT de la imagen capturada. Este paso dará como resultado un conjunto de vectores numéricos. La cardinalidad de dicho conjunto varía de una imagen a otra.

- $\quad$ Se compara cada uno de los descriptores del paso anterior con los almacenados previamente en una base de datos. Los descriptores almacenados corresponden a especies de serpientes conocidas por lo que cada descriptor comparado podrá ser contabilizado como perteneciente a determinado tipo de serpiente.

- $\quad$ Luego, se identificará la imagen del ejemplar ingresado como perteneciente a la especie que mayor cantidad de coincidencias presente.

Un detalle importante del proceso anterior es que puede utilizarse la cantidad de coincidencias detectadas para determinar el grado de confianza de la predicción.

Base de Datos de serpientes

La base de datos de serpientes está formada por los descriptores SIFT de ejemplares de las distintas especies que habitan la región de interés. Las imágenes fueron obtenidas, en su mayoría, en ambientes naturales. En su selección, se tuvo una consideración especial con distintas variantes como:

- El ciclo en el que se encuentra el ejemplar: como se mencionó en la introducción, las serpientes mudan de piel. En esta etapa, esta luce más opaca, lo que implica cambios críticos al momento de obtener los descriptores de las imágenes.

- $\quad$ El contexto de hallazgo del ejemplar: cabe destacar que, dependiendo de la condición climática, la piel de animal se verá distinta. Por ejemplo, en un lugar húmedo, la piel será más brillosa que en una zona rocosa, donde probablemente presente vestigios de tierra.

- $\quad$ La postura del ejemplar: no siempre se puede encontrar al animal estirado. En muchas situaciones, el mismo se encuentra enrollado en sí mismo o en una rama.

- $\quad$ Como se dijo anteriormente, un ejemplar puede ser encontrado en pastizales, roquedas, etcétera. En estos casos, el entorno nunca es controlado, sino que en algunas situaciones puede ser muy complejo (por ejemplo, en zonas acuáticas rodeadas de pastos) y ocultando distintas partes del animal.

Por los motivos mencionados, y siguiendo las recomendaciones del asesor profesional del CEPAVE, se seleccionaron y recortaron manualmente aquellas regiones distintivas de las especies a estudiar. De esta manera, se descartó el fondo y el ruido de cada segmento. En la figura 2 se observan algunos recortes que forman parte de la base de datos. Cabe destacar que en todas las imágenes se manejó un nivel de escala que 


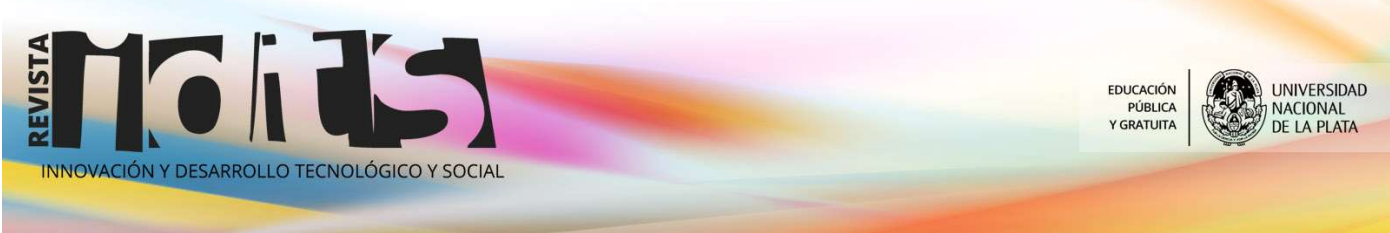

Innovación y Desarrollo Tecnológico y Social (2019) 1 (1): 41-51

permite distinguir los dibujos característicos de cada especie. Por lo tanto, la base de datos quedó constituida por los conjuntos de descriptores SIFT de cada uno de estos recortes.
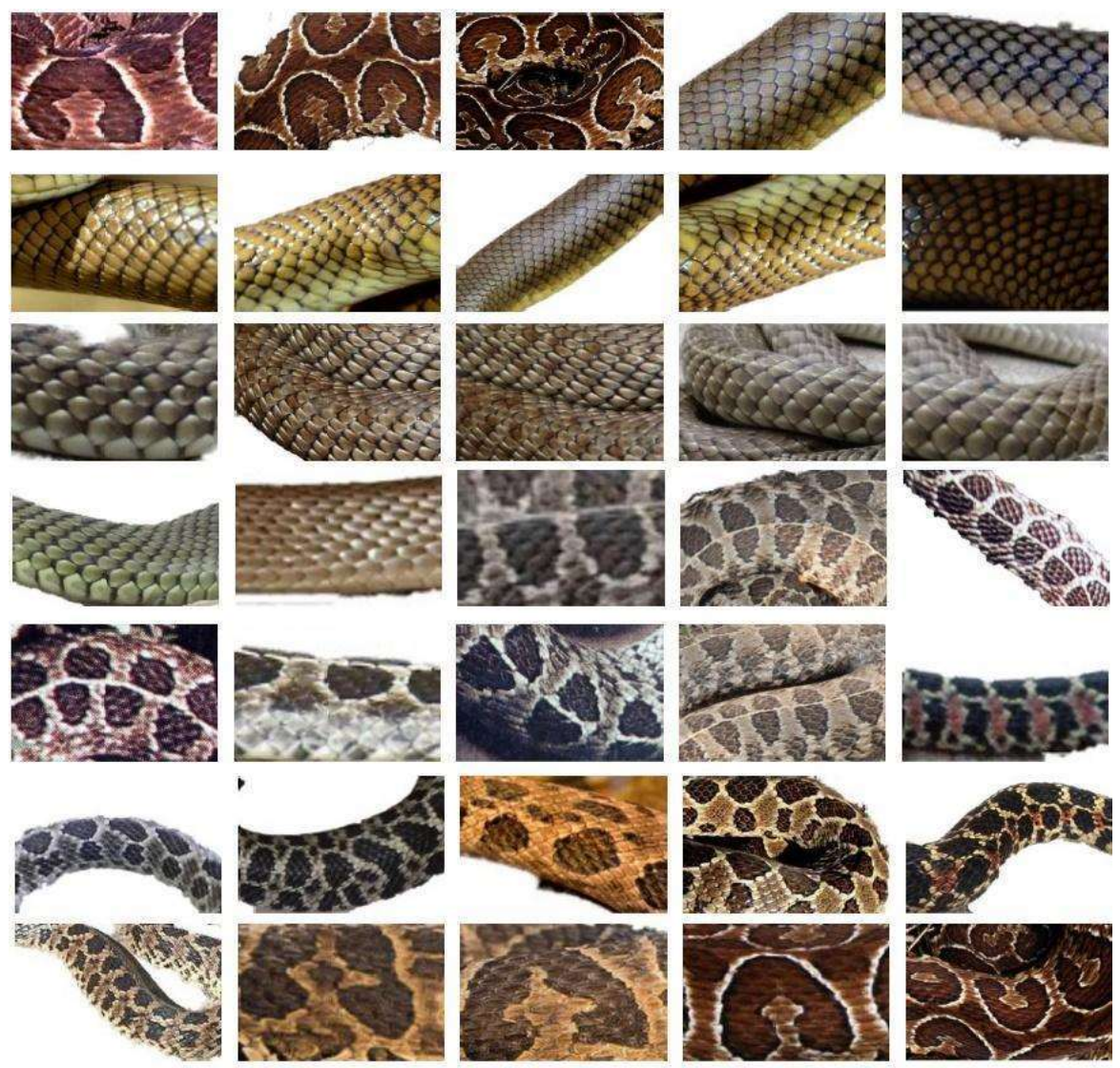

Figura 2. Recortes de cuerpos de serpientes donde se pueden observar los patrones que caracterizan a cada ofidio / Figure 2. Cuttings of snake bodies where the characteristic patterns of each ophidium can be observed

Una vez construida la base, para clasificar un nuevo ejemplar se debe comparar cada uno de los descriptores que componen la imagen suministrada con los descriptores de los recortes de la base. Es importante considerar que un mismo descriptor puede ser asociado a varios recortes, especialmente porque en la base de datos se han utilizado las distintas partes de un mismo ejemplar como zona de interés. Incluso puede ocurrir que un mismo descriptor sea asociado a distintos tipos de serpiente ya que podría ocurrir que tuvieran características comunes. Al repetir este proceso para todos los descriptores de la imagen a clasificar se obtendrán las cantidades de coincidencias para cada especie. La especie con mayor cantidad de coincidencias será la utilizada para dar la respuesta.

Para asociar un descriptor a un recorte debe cumplirse que su distancia al descriptor más cercano dentro de dicho recorte sea significativa. Para ello, se toman las dos distancias a los dos descriptores del recorte más cercanos al descriptor a analizar. Sean D1 y D2 esas distancias siendo D1 menor que D2. Luego se considera que la distancia 


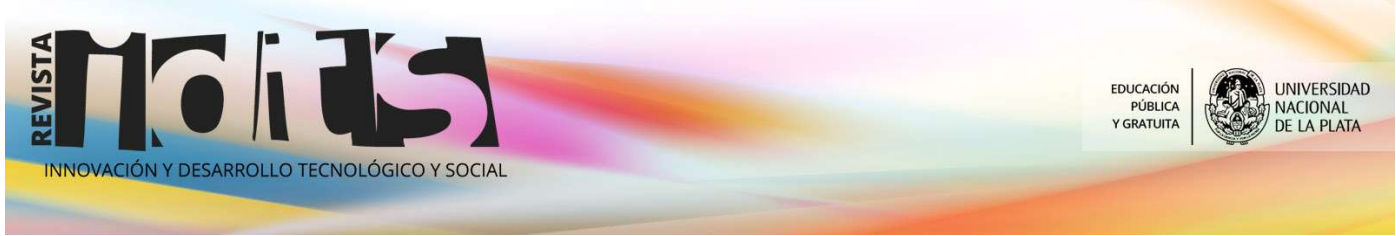

Innovación y Desarrollo Tecnológico y Social (2019) 1 (1): 41-51

al descriptor más cercano dentro de dicho recorte, D1, es significativa si el cociente D2/D1 es superior a un cierto valor umbral. En resumen el umbral es una herramienta para ponderar la magnitud de la diferencia entre ambas distancias y debe ser definido a priori.

Dado que el valor de umbral a utilizar juega un papel muy importante en la precisión del clasificador se realizó un estudio detallado del grado de participación de cada descriptor de la base de datos en la predicción de la especie a la que pertenece. Utilizando un proceso de validación cruzada se trabajó con distintos umbrales para establecer cuál era el valor con mejor desempeño.

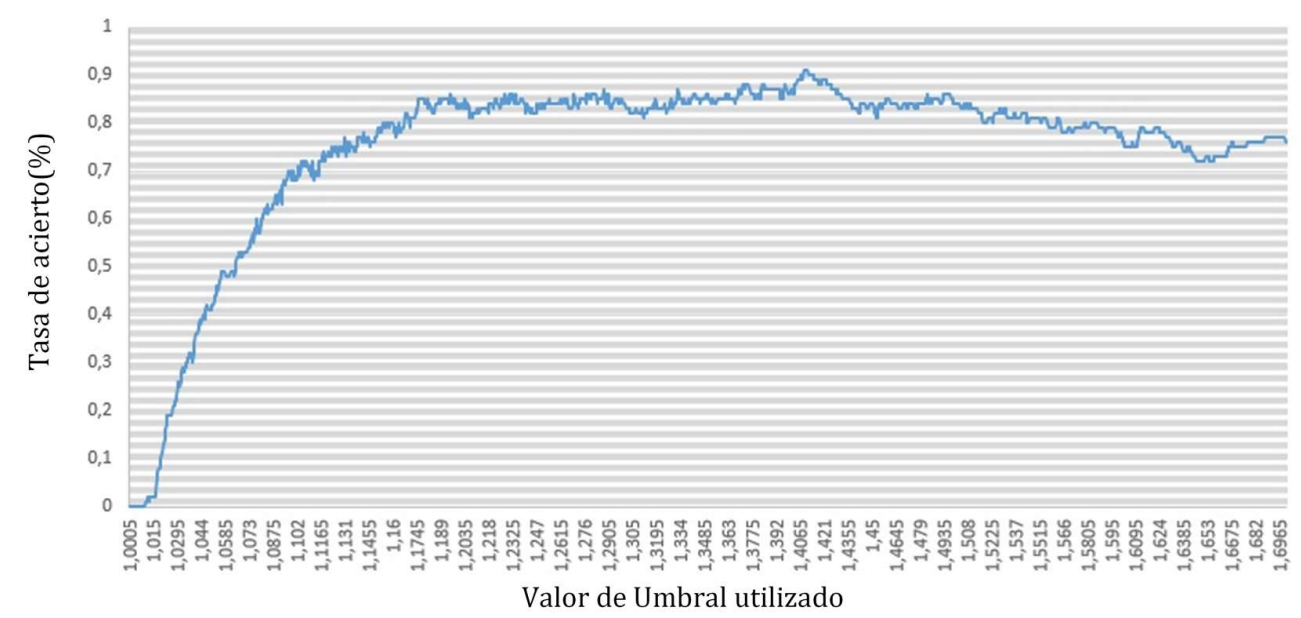

Figura 3. Tasa de acierto obtenida para cada valor de umbral / Figure 3. Accuracy obtained for each threshold value

Tal como se observa en la Figura 3 la mejor tasa de acierto es del $91 \%$ al utilizar como valor de umbral 1,4080. Por lo tanto, éste fue el valor utilizado por el reconocedor para decidir el parecido de un descriptor con los descriptores de cada imagen de la base.

Para probar el desempeño del modelo se efectuó una validación cruzada utilizando $\mathrm{k}=10$ conjuntos de imágenes. Cada conjunto estuvo formado a su vez por 10 imágenes. Se realizaron 30 ejecuciones independientes utilizando dos formas de selección distintas: aleatoria y estratificada. En la selección aleatoria no se efectúa ningún control sobre la proporción de imágenes por especies mientras que en la selección estratificada se verifica que cada uno de los 10 conjuntos contenga la misma proporción de imágenes por especie que el conjunto completo. La Figura 4 muestra los resultados obtenidos en cada una de las ejecuciones. Si bien ambos casos tuvieron un buen desempeño, tal como era de esperar, la selección estratificada ofreció una tasa de acierto superior. 


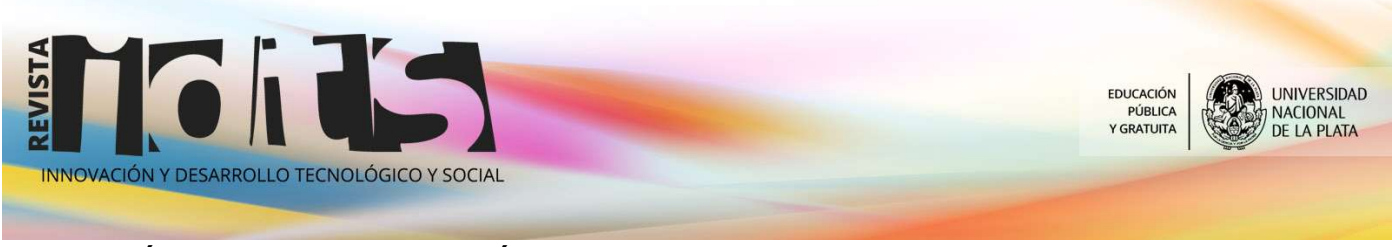

Innovación y Desarrollo Tecnológico y Social (2019) 1 (1): 41-51

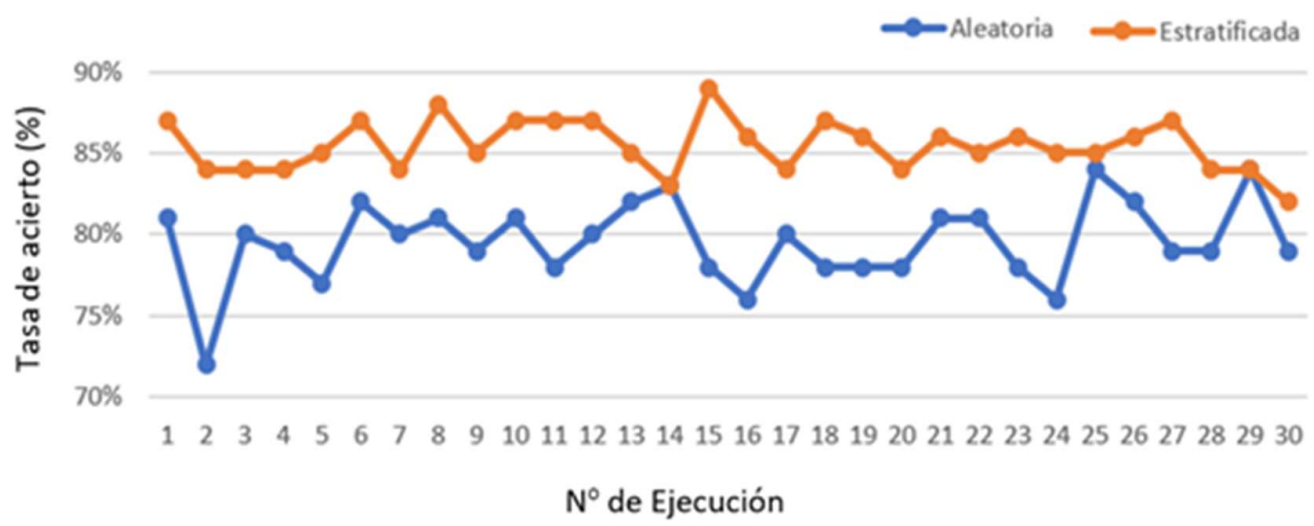

Figura 4. Aciertos obtenidos en cada una de las 30 ejecuciones independientes. / Figure 4. Successes obtained in each of the 30 independent executions.

Finalmente, las Figuras 5 y 6 muestran los resultados obtenidos en la clasificación de dos ejemplares de cada una de las cinco especies más populares en el partido de La Plata y Gran La Plata. Las especies analizadas fueron las siguientes: Bothropus Alternatus, Paraphimophis Rusticus. Philodryas Patagoniensis, Tomodon Ocellatusy Xenodon Dorbignyi.

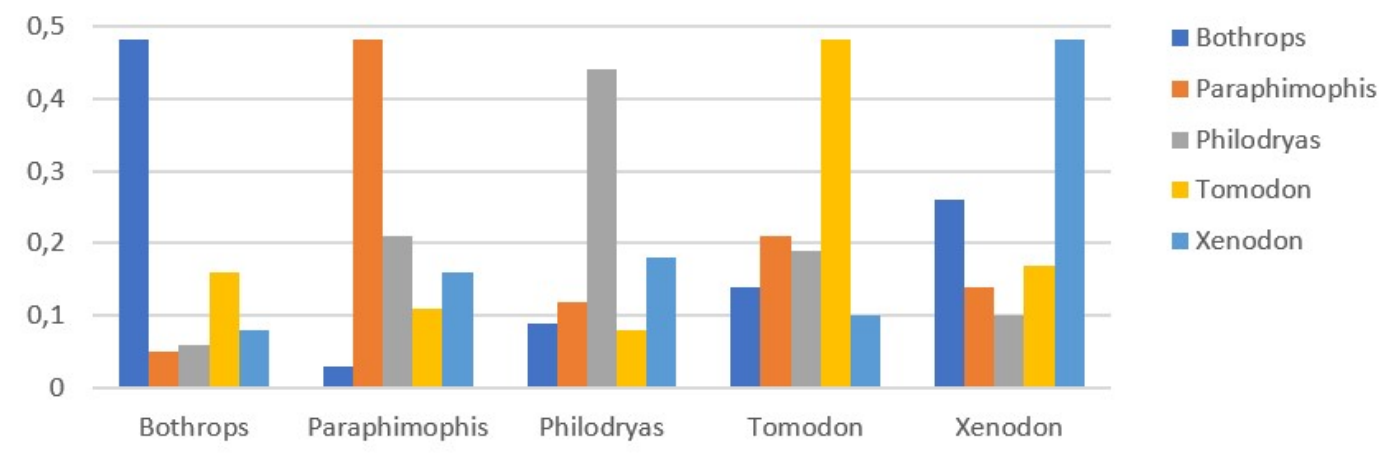

Figura 5. Reconocimiento de ejemplares utilizando el modelo propuesto. / Figure 5. Recognition of specimens using the proposed model. 


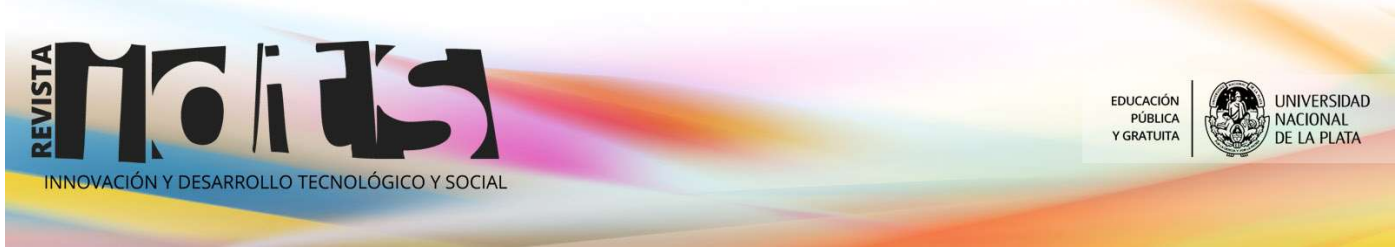

Innovación y Desarrollo Tecnológico y Social (2019) 1 (1): 41-51

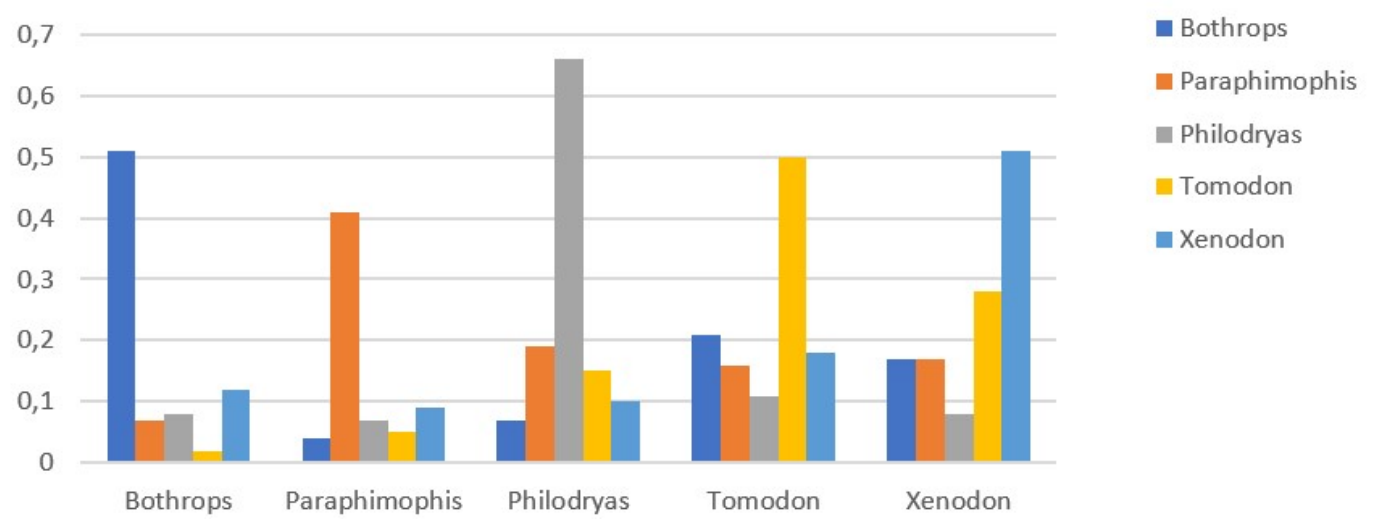

Figura 6. Segunda prueba de reconocimiento/ Figure 6. Second recognition test

En estas figuras pueden verse los diagramas de barras correspondientes a las asignaciones de los descriptores que componen cada imagen a reconocer en las especies que se encuentran representadas por los recortes de la base. Los diez diagramas de barras de las Figuras 5 y 6 muestran la existencia de características comunes entre las especies. Sin embargo, existe una especie mayoritaria fácilmente identificable.

Por otro lado, las Figuras 5 y 6 permiten observar que esas características comunes no se encuentran siempre presentes entre las imágenes de distintos ejemplares. Esto queda evidenciado por la distribución de frecuencias de los diagramas de la Figura 5 con respecto a los de la Figura 6.

Observando las Figuras 5 y 6 puede afirmarse que, en todos los casos se observa que el modelo propuesto reconoce correctamente los ejemplares ingresados.

\section{Conclusiones y trabajos futuros}

El reconocimiento de objetos en imágenes puede aplicarse en distintos contextos. Existen soluciones previas desarrolladas por algunos de los autores de este trabajo aplicadas al reconocimiento de rostros como medida biométrica (Lanzarini et al., 2013). En todos los casos, la dificultad reside en la construcción adecuada de la base de datos de imágenes con las que se compararán los objetos (ejemplares de serpientes en este caso) a reconocer. Esto implica determinar la representación a utilizar. En este caso se decidió emplear los descriptores SIFT y se comprobó que su desempeño era adecuado para la resolución del problema planteado.

Los resultados de esta investigación fueron presentados en (La Frazia et al., 2018) y difundidos a través del sitio CONICET-La Plata (CONICET, 2018).

Si bien los reconocimientos realizados hasta el momento han arrojado resultados satisfactorios quedan algunos aspectos por resolver, entre los que se encuentra la necesidad de definir una clase de rechazo. El reconocedor propuesto utiliza la proporción de casos asignados a la clase mayoritaria como instrumento para medir el grado de acierto de la respuesta dada. La aplicación siempre responderá que el ejemplar pertenece a alguna especie, aunque su grado de certeza sea bajo. Queda a criterio del usuario el ingresar una nueva imagen para obtener una certeza mayor. 


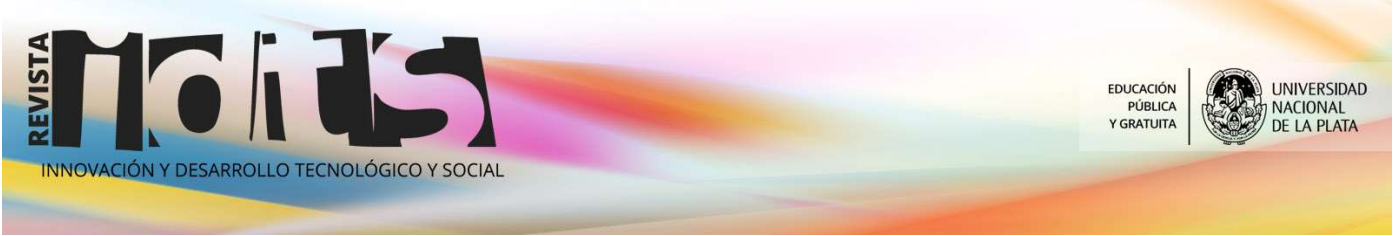

Innovación y Desarrollo Tecnológico y Social (2019) 1 (1): 41-51

Queda pendiente extender este desarrollo a todas las especies de la provincia de Buenos Aires.

\section{Referencias bibliográficas}

Amiza A., Zahri B., Hanin N, Naimah Y., Badlishah A. (2017). Image Classification for Snake Species Using Machine Learning Techniques. Proceedings of the Computational Intelligence in Information Systems Conference (CIIS 2016) pp 52-59.

CONICET (2018). CEPAVE, CONICET-UNLP-ASOCIADO A CICPBA. Desarrollan una herramienta digital que permitirá identificar los tipos de serpientes. Recuperado de https://laplata.conicet.gov.ar/desarrollan-una-herramientadigital-que-permitira-identificar-los-distintos-tipos-de-serpientes/

Giambelluca, L. (2015). Serpientes bonaerenses. Editorial EDULP, La Plata. Bs.As. Argentina.

La Frazia L, Lanzarini L, Estrebou C y Giambelluca L. (2018). Reconocimiento de serpientes del partido de La Plata mediante un método informático. XIX Congreso Argentino de Herpetología. Octubre de 2018. U.N.L.P.

Lanzarini L., Ronchetti F., Estrebou C., Lens L. y Fernández Bariviera A. (2013). Face recognition based on fuzzy probabilistic SOM, 2013 Joint IFSA World Congress and NAFIPS Annual Meeting (IFSA/NAFIPS), Edmonton, AB, 2013, pp. 310-314.

Lowe D. (1999). Object recognition from local scale-invariant features.

Computer Vision, 1999. Proceedings of the Seventh IEEE International Conference on, vol.2, pp.1150-1157 1999 doi: 10.1109/ICCV.1999.790410

Lowe D. (2004). Distinctive Image Features from Scale-Invariant Keypoints. Int. J. Comput. Vision. Volumen 60, nro. 2, pp.91-110. 2004. 